\title{
Child mortality and water stress under the framework of Sustainable Development Goals (SDGs): Evidence from twenty developing countries
}

\section{Suyu Liu}

Green Growth Knowledge Partnership (GGKP), United Nations Environment Programme (UNEP), Geneva, Switzerland; e-mail: suyu.liu@linacre.ox.ac.uk

Keywords: child mortality; water stress; Sustainable Development Goals (SDGs); path analysis; cross-country comparison

\begin{abstract}
Both child mortality and water stress are included into the Sustainable Development Goals (SDGs). Existing studies have explored the relationship between child mortality and water stress with different perspectives, however, the results vary due to differences in methods and context. There is still insufficient cross-country comparative research on the association between child mortality and water stress under the framework of SDGs. This article uses data of 20 developing countries to explore the association between child mortality and water stress, based on the path analysis methodology. The results show that in term of statistics, the level of water stress is negatively but insignificantly associated with child mortality in developing countries. This unconventional result is explained by a number of possible reasons such as high reliance on food importation in some developing countries, and also the indirect link between water stress and child mortality via a country's socioeconomic development (measured by national poverty rate). The results enrich scientific knowledge of and provide practical implications to the interactions between SDGs.
\end{abstract}

\section{Introduction}

There is a sustained scientific and policy interest in the availability of water resources and the management of water, including water-use efficiency (e.g., Bauer et al, 2020). An indicator widely-used to measure the availability of water resources is the level of water stress, which has different definitions at both qualitative and quantitative dimensions. For example, Kummu et al $(2016$, p2) define water stress as '...the impact of high water use (either withdrawals or consumption) relative to water availability'. Under the framework of the United Nations (UN) Sustainable Development Goals (SDGs), the World Bank defines the level of water stress as 'ratio between total freshwater withdrawal by all major sectors and total renewable freshwater resources' (World Bank, 2021), which has been formally accepted as an indicator (SDG indicator 6.4.2) to measure the progress of achieving SDGs. This definition has the advantage of relatively easy to measure and high availability of data, which are important reasons for its inclusion as a global level indicator. Also environmental water requirements are incorporated into this concept.

Child mortality also attracts attentions from researchers and practitioners. A widely selected indicator to measure child mortality is the under- 5 mortality rate, which refers to 
the amount of (predicted) deaths per 1,000 children before reaching age five. This concept is rather straightforward which seldom causes misunderstanding. Its simplicity and wide adaptation become important reasons for its inclusion as an SDG indicator (3.2.1).

This article explores the relationship between SDG indicator 3.2.1 'under-five mortality rate' and SDG indicator 6.4.2 'level of water stress' (United Nations, 2021), with the perspective that different SDGs (and also different SDG indicators) are interactive with each other (e.g., Liu, 2020). Previous studies have explored the association between child mortality and water stress from different perspectives. A typical link between child mortality and water stress is that unavailability of water may lead to negative health impact on children, which may result in higher child mortality. For example, Bizuneh et al (2017) and He et al (2018) use Ethiopia and Nigeria as case studies to show that lack of access to water may lead to higher probability of diseases in children, which is an important reason of child mortality. However, based on panel data of 59 countries, Headey and Palloni (2019) find that access to water is not significantly related to children's health, with an exception that installation of piped water into households may reduce child stunting. Another cross-national study of 41 countries demonstrates that child mortality is not significantly with water supply, although unimproved water supply is harmful to children's health (Geere and Hunter, 2020).

Water stress also affects child mortality as the former has a direct impact on food security, which is related to nutrition and child mortality. For example, Suárez-Varela et al (2018) argue that water is essential for food production, processing, and preparation. In the specific case of Morocco, water scarcity is a challenge which needs to be addressed in order to underpin food security (Suárez-Varela et al, 2018). Similar situations take place in many other countries especially the developing world (e.g. Qiu et al, 2012), where agriculture is more resource-extensive and thus require large amounts of water supply. Vanham and Leip (2020) also argue that properly addressing the challenge of water stress is essential for a sustainable food system. Given the widely-perceived connection between hunger and death of children (Lucas et al, 2019), it is able to understand that water stress is a factor which may lead to child mortality.

Water stress is strongly associated with sanitation and hygiene services, which is also a reason of its connection with child mortality. For example, with an Ordinary Least Squares (OLS) analysis, Geere and Hunter (2020) demonstrate that the use of sanitation facilities would increase health outcomes among children. Similarly, Headey and Palloni (2019) find that although improvements in sanitation may not significantly reduce child stunting and wasting, they can reduce child mortality and widely-observed fatal diseases such as diarrhea. Although Gera et al (2018) do not find robust evidence to support water, sanitation, and hygiene's (WASH) strong impact on child mortality, they realize the potential health benefits of WASH and support the provision of adequate access to WASH. In fact, water is very substantially influenced by, and also has strong influence on sanitation and hygiene, that is a reason why these three terms are often mentioned and abbreviated together.

Further to these more direct links between water stress and child mortality, scholars also find water stress is related to socioeconomic development, or sometimes is part of 
socioeconomic development, and therefore can affect child mortality in an indirect approach. For example, Huang et al (2019) show that water-use efficiency and relevant technology can have an impact on water stress. Access to such technology may be unequal which often results in depletion of (water) resources and creation of poverty and poverty gaps (Mirza et al, 2019), and poverty is widely-known for its influence on child mortality. Adams et al (2020) illustrate that water stress affects more on the mortality of poor people (including children) living in the Global South. For example, poor people living in the Global South may not have adequate access to water and therefore need to physically fetch water with heavy containers, which leads to injuries and deaths (including children).

Existing literature has contributed to knowledge and practice enormously. However, even though water stress and child mortality have been included into the SDGs, previous studies touch less on their relationship with the perspective of SDGs, including the interactions between different SDGs and also different SDG indicators. In addition, in comparison with in-country case studies, cross-country comparisons related to this topic is still insufficient, especially in the developing context. Practical implications and the applicability of policy recommendations are also reduced due to the above-mentioned reasons.

Therefore, this article aims to enrich the scientific and practical knowledge to bridge these gaps. By using the data from twenty developing countries (low-income and lower-middle-income countries as classified by the World Bank), this article has the objective to generate more cross-national knowledge and implications. Exploration on the connections between water stress and child mortality under the framework of SDGs would enable the article to explore more about the interaction between different SDGs (as well as different SDG indicators). Such interactions may also assist policy-makers to properly balance and prioritize different SDGs (and also SDG targets). Furthermore, by adding path analysis, this article would extend the in-depth analysis on the mechanism of how water stress affect child mortality.

The remaining parts of the article will be arranged as followed. The next section will introduce the research methodology and the data. The section after the next one will summarize the empirical results and discuss the results, with special emphasis on the interactions of different SDGs. A number of practical implications will also be provided based on the results. In the conclusion, the main contributions of the article will be mentioned, and the shortcomings are also acknowledged, which will provide spaces for future research in this field.

\section{Methodology and data}

\subsection{Methodology}

This article uses OLS to analyze the relationship between level of water stress and child mortality. OLS is a tool to identify the strength of relationship between a responsive variable and one or more explanatory variables, based on the least-squares technique to minimize the deviation from the best-fit line. This is a common method which has been used to identify whether and how child health is attributable to environmental factors 
(e.g., Doeffinger and Hall, 2020; Shaw et al, 2020). This is possibly because of its convenience when applying in relatively simpler models (e.g., with fewer variables, without the objective to identify causal relationships). Since other socioeconomic factors also affect child mortality, this article chooses 'percentage of population living below the national poverty line' (United Nations, 2021) as an instrument variable to reflect socioeconomic factors of a country (in this article, it appears in the name 'national poverty rate'). This is not only because of the consensus that poverty is associated with economic development, income level, and technology progress (Mirza et al, 2019), but also because of the easy access to the data. It is also an SDG indicator (1.2.1), which enables a better analysis of interactions between SDGs.

Furthermore, as shown in the previous literature, water stress may also affect child mortality indirectly such as via socioeconomic factors, this article also conducts a path analysis to test this indirect means. The whole path can be shown in Figure 1 as below. That means, as shown by the arrows in Figure 1, water stress can affect child mortality directly, and also indirectly via its impact on national poverty. National poverty can also affect child mortality. The possible endogeneity problem, such as the possibility that the national poverty rate can cause the changes of level of water stress, is excluded by the differences in the year of data for different variables. In other words, something happened latter cannot be the causal reasons of something happened earlier. Therefore, in this article the data for national poverty and child mortality are later than data for water stress. That is why in Figure 1 there is no arrow pointing at the level of water stress.

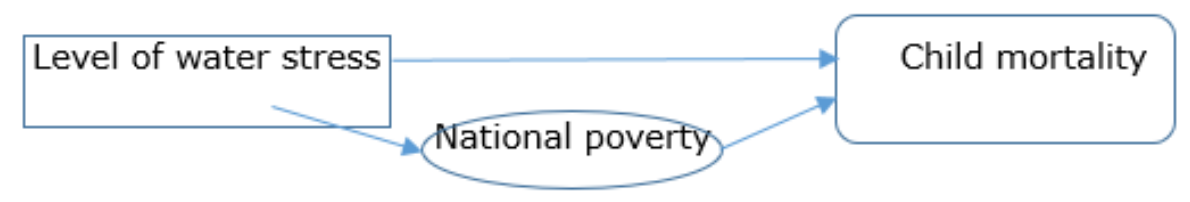

Figure 1. Path analysis of water stress and child mortality

The standard OLS regression is written as:

Child mortality $=b_{0}+b_{1}$ (Level of water stress) $+b_{2}$ (National poverty) $+e$, where $b_{0}, b_{1}, b_{2}$ are coefficients and e is the error term.

The path analysis model is further presented as below, where $c_{0}, d_{0}$, and $f_{0}$ are constants, $c_{1}, d_{1}$, and $f_{1}$ are coefficients, and $e_{1}, e_{2}$ and $e_{3}$ are relevant error terms. The path analysis will show the beta values of the coefficients.

Child mortality $=\mathrm{C}_{0}+\mathrm{c}_{1}$ (Level of water stress) $+\mathrm{e}_{1}$

Child mortality $=\mathrm{d}_{0}+\mathrm{d}_{1}$ (National poverty) $+\mathrm{e}_{2}$

National poverty $=f_{0}+f_{1}$ (Level of water stress) $+e_{3}$

\subsection{Data}

Data of the level of water stress in the twenty selected developing countries are the percentages of freshwater withdrawn in the total available freshwater resources in each country. The twenty countries are selected not only because of geographic representativeness, but also due to the availability of data. All these figures are for the year 2014, which is also the most recent year when the level of water stress data is available. The under-five mortality rate data are all for the year 2019 (most recent). However, in the data source the figures are reported as per 1,000, and for the convenience of analysis and calculation, in this article the figures are converted into 
percentages with division by 10 . Data of poverty rate in these countries are for the year between 2015 and 2018 (inclusive), so that they will not cause problems related to endogeneity, as something happened later cannot become reasons of something happened earlier. In this case, the child mortality in 2019 cannot become reasons for poverty rates between 2015 and 2018, and poverty rates in 2015 or later cannot become reasons of level of water stress in 2014. The data are shown in the Table 1 below.

Table 1. The dataset

\begin{tabular}{lccc}
\hline \multicolumn{1}{c}{ Country } & $\begin{array}{c}\text { Level of Water } \\
\text { stress (\%) }\end{array}$ & $\begin{array}{c}\text { National poverty } \\
\text { rate (\%) }\end{array}$ & $\begin{array}{c}\text { Child mortality } \\
\text { rate (\%) }\end{array}$ \\
\hline Afghanistan & 43.67 & 54.5 & 6 \\
Angola & 0.68 & 32.3 & 7.5 \\
Bangladesh & 3.79 & 24.3 & 3.1 \\
Bhutan & 0.56 & 8.2 & 2.9 \\
Burkina Faso & 9.49 & 41.4 & 8.8 \\
Chad & 2.44 & 42.3 & 11.4 \\
Eswatini & 32.35 & 58.9 & 4.9 \\
Ethiopia & 11.6 & 23.5 & 5.1 \\
Gambia & 1.45 & 48.6 & 5.2 \\
Lao PDR & 1.39 & 18.3 & 4.6 \\
Malawi & 11.08 & 51.5 & 4.2 \\
Moldova & 13.22 & 23 & 1.4 \\
Mongolia & 2.43 & 28.4 & 1.6 \\
Nicaragua & 1.35 & 24.9 & 1.7 \\
Niger & 3.75 & 40.8 & 8 \\
Nigeria & 5.83 & 40.1 & 11.7 \\
Rwanda & 1.44 & 38.2 & 3.4 \\
Tanzania & 7.45 & 26.4 & 5 \\
Togo & 1.77 & 55.1 & 6.7 \\
Zambia & 2.14 & 54.4 & 6.2 \\
Mean & 7.89 & 36.8 & 5.5 \\
(simple) & & & \\
\hline (Data source: World Bank Open Data https://data.worldbank.org/ accessed 20 January 2021) &
\end{tabular}

\section{Results and discussion}

\subsection{Results}

The regression results are shown in Table 2 below.

Table 2. Regression results

\begin{tabular}{lccc}
\hline Dependent variable & Coefficients & Standardized coefficients (SD) & R Square \\
\hline Child mortality & $\mathrm{b}_{0}=2.02$ & & 0.223 \\
& $\mathrm{~b}_{1}=-0.06$ & $\mathrm{SDb}_{1}=0.062$ & \\
& $\mathrm{~b}_{2}=0.107^{*} *$ & $\mathrm{SDb}_{2}=0.048$ & 0.00 \\
Child mortality & $\mathrm{C}_{1}=-0.006$ & $\mathrm{SDC}_{1}=0.062$ & 0.181 \\
Child mortality & $\mathrm{d}_{1}=0.425^{*}$ & $\mathrm{SDd}_{1}=0.044$ & 0.181 \\
National poverty & $\mathrm{f}_{1}=0.425^{*}$ & $\mathrm{SDf}_{1}=0.274$ &
\end{tabular}

('**' and '*' symbolize the statistical significance at 0.05 and 0.1 levels respectively)

The results of path analysis can also be shown Figure 2 as following: 


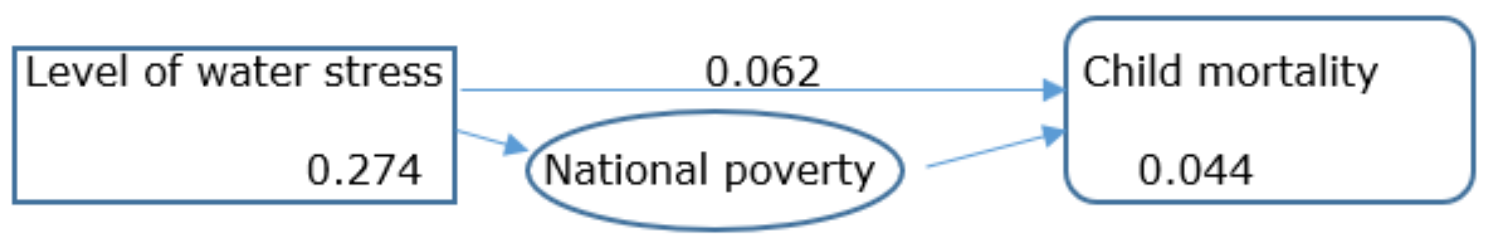

Figure 2. Path analysis results

For the standard OLS regression, the results show that the level of water stress is negatively associated with child mortality in developing countries. As reflected by the value of $b_{1}$, a one percentage point increase in the level of water stress, which mean a one percentage point decrease of the un-withdrawn freshwater resource will lead a reduction of 0.06 percentage point in child mortality. However, this value is statistically insignificant. A one percentage increase in national poverty rate will lead to an increase of child mortality by near 0.11 percentage point, and this is also significant in term of statistics.

The beta values of the coefficients which are used for the path analysis also support the above findings. For example, level of water stress is very weakly associated with child mortality at country level, and the association is negative but insignificant in statistics. The R Square value $(0.00)$ also shows that the level of water stress has very weak explanatory power to the differences in child mortality. To the contrary, child mortality rate is positively and significantly associated with national poverty rate. The beta value of $d_{1}$ and $f_{1}$ also suggest that the water stress's influence on child mortality is almost completely through its impact on national poverty, while the direct impact is very weak.

\subsection{Discussion}

The statistical results in the previous section is beyond the traditional knowledge to some extent. The very weak and negative association between level of water stress and child mortality in developing countries is not consistent with previous studies in the introduction, which often find that the higher availability of water resources is contributory to the reduction of child mortality. A main reason to this unconventional result could be that the food production in some developing countries has no strong reliance on the water availability, as these countries import substantial amounts of food to satisfy the domestic demands (e.g., Ewing and Msangi, 2009).

This finding is also possibly due to the differences in water-use efficiencies across countries. Water-use efficiency, which is often reflected by the value of outputs per cubic meter of water used, is another SDG indicator (6.4.1) but not explored in this article. Cole et al (2018) find that socioeconomic factors such as income rather than natural factors such as precipitation affect more on water use including water-use efficiency. Bauer et al (2020) also suggest that water-use efficiency including the reuse of water is associated with levels of technology, that is another important restriction for several developing countries which usually have insufficient access to advanced technologies. 
It is not beyond the popular belief that the level of water stress, which is a negative indicator of water availability, is positively linked with national poverty in developing countries. For example, this finding lends support to Baah-Kumi and Ward (2020), which argues that in developing countries such as Ghana and Bukina Faso, infrastructure such as water reservoirs can mitigate poverty. This is particularly in the context where water availability fluctuates sharply between wet seasons/years and dry seasons/years (Baah-Kumi and Ward, 2020), which may also be an important reason of the seasonal/annual fluctuations in economic performance and incomes in developing countries.

The findings also support that interactions exist among different SDGs (e.g., Nilsson et al, 2006). For example, the relationship between poverty reduction (SDG Target 1.2) and child mortality (SDG Target 3.1) are demonstrated in this article. In addition, via the discussion with previous literature, the article also finds that SDGs related to water and sanitation (such as SDG 6) has interactions with SDGs about food, health, and infrastructure (such as SDGs 2, 3, and 9). Such interactions are complicated and vary across countries, which require further exploration in future. This is particularly the situation for some developing countries, which have relatively weak statistical capacities as reflected by the SDG Target 17.19.

A number of practical implications and policy recommendations can be generated from this article. Firstly, since health outcomes including child mortality is strongly associated with national poverty rate which reflects the overall socioeconomic development, economic growth should still be prioritized in the national development strategies in these countries. Environment is certainly an important dimension of economic development, and it should be incorporated rather than excluded from national development strategies. Therefore, a more balanced approach with suitable prioritization of economic growth would be essential for many developing countries. Secondly, country-specific context must be considered in order to generate suitable evidence-based policies to achieve economic, health, and environmental outcomes. For example, it is necessary to identify a country's technological level before exploring the association between water use and child mortality. Thirdly, statistical capacity in developing countries should be improved, which is essential to make well-informed developmental policies based on solid empirical evidence. This can also benefit future research in this field.

\section{Conclusions}

This article uses empirical evidence to explore the relationship between water stress and child mortality in twenty developing countries. National poverty rate is also selected as a control variable as it is a suitable compound variable to reflect the overall situation in the developing countries. The results suggest that the level of water stress is very weakly associated with child mortality, but instead, national poverty rate is positively and significantly associated with child mortality. In addition, as shown in the path analysis, level of water stress's influence on child mortality is almost fully via the indirect means. This lends some support to existing literature. 
This article supports the research on the interactions between SDGs (including different SDG indicators). For example, the empirical evidence enriches the knowledge of the connections between SDGs about health, environment, and economic growth. Via further discussion with previous studies, the article contributes to explore whether and how different SDGs, especially those strongly related to health, poverty, environment, and infrastructure are interrelated to each other. This can generate suitable evidence to support well-informed and more balanced national development strategies, which must be considered in line with the national and regional contexts.

The article is of course not without limitations. One shortcoming is the unavailability of data, which is a core reason why only these twenty developing countries are selected. Insufficient over-year data excludes the possibility to conduct panel data analysis. Another weakness is that the article cannot incorporate more variables from the SDGs, due to the limited number of data and countries selected. This also reduces the article's capacity to explore more on the interactions between SDGs. This also suggests that there is still a long way forward to achieve the SDG Targets 17.18 and 17.19 , which call for further improvements of data capacity. Future studies may also explore the relationship between water stress and child mortality in more developed countries, which may have better access to advanced technologies and healthcare services. A comparison between the situation in developed and developing countries may also generate interesting findings and provide evidence for more comprehensive policies to achieve the SDGs.

Acknowledgements: The author is grateful to the insights of (former) colleagues and peers at the United Nations (UN) including the United Nations Environment Programme (UNEP). However, unless otherwise specified, the views expressed in this article are the authors and do not reflect any official stance of the UN and any organization related to it, including the UNEP.

\section{References}

1. Adams, E.A., Stoler, J. and Adams, Y. (2020) Water insecurity and urban poverty in the Global South: Implications for health and human biology. American Journal of Human Biology. 32: e23368. https://doi.org/10.1002/ajhb.23368

2. Baah-Kumi, B. and Ward, F.A. (2020) Poverty mitigation through optimized water development and use: Insights from the Volta Basin. Journal of Hydrology. 582: 124548. https://doi.org/10.1016/j.jhydrol.2020.124548

3. Bauer, S., Dell, A., Behnisch, J. et al (2020) Water-reuse concepts for industrial parks in water stressed regions in South East Asia. Water Supply. 20: 296-306. doi: 10.2166/ws.2019.162

4. Bizuneh, F., Getnet, F., Meressa, B. et al (2017) Factors associated with diarrheal morbidity among under-five children in Jigjiga town, Somali Regional State, eastern Ethiopia: a cross-sectional study. BMC Pediatrics. https://doi.org/10.1186/s12887-017-0934-5

5. Cole, M.J., Bailey, R.M., Cullis, J.M.S. (2018) Spatial inequality in water access and water use in South Africa. Water Policy. 20: 37-52. doi: https://doi.org/10.2166/wp.2017.111

6. Doeffinger, T. and Hall, J.W. (2020) Water stress and productivity: An empirical analysis of trends and drivers. Water Resources Research, 56: e2019WR025925. https://doi.org/10.1029/2019WR025925

7. Ewing, M., \& Msangi, S. (2009). Biofuels production in developing countries: assessing tradeoffs in welfare and food security. Environmental Science \& Policy, 12(4), 520-528. https://doi.org/10.1016/j.envsci.2008.10.002 
8. Geere, J-A. L. and Hunter, P.R. (2020) The association of water carriage, water supply and sanitation usage with maternal and child health. A combined analysis of 49 Multiple Indicator Cluster Surveys from 41 countries. International Journal of Hygiene and Environmental Health. 223(1): 238-247. https://doi.org/10.1016/j.ijheh.2019.08.007

9. Gera, T., Shah, D. and Sachdev, H.S. (2018) Impact of water, sanitation and hygiene interventions on growth, non-diarrheal morbidity and mortality in children residing in lowand middle-income countries: a systematic review. Indian Pediatrics. 55:381-431. https://pubmed.ncbi.nlm.nih.gov/29428924/

10. He, Z., Bishwajit, G., Zou, D. et al (2018) Burden of common childhood diseases in relation to improved water, sanitation, and hygiene (WASH) among Nigerian children. International Journal of Environmental Research and Public Health. 15: 1241. http://dx.doi.org/10.3390/ijerph15061241

11. Headey, D. and Palloni, G. (2020) Water, sanitation, and child health: evidence from subnational panel data in 59 countries. Demography. 56:729-752. https://doi.org/10.1007/s13524-019-00760-y

12. Huang, H., Wang, J., Han, Y. et al (2019) Assessing impacts of water regulations on alleviating regional water stress with a system dynamics model. Water Supply. 19(2): 635-643. https://doi.org/10.2166/ws.2018.112

13. Hutcheson, G. D. (1999). The multivariate social scientist. SAGE Publications, Ltd. https://www.doi.org/10.4135/9780857028075

14. Kummu, M., Guillaume, J.H.A., de Moel, H. et al (2016) The world's road to water scarcity: shortage and stress in the 20th century and pathways towards sustainability. Scientific Reports 6: 38495. https://doi.org/10.1038/srep38495

15. Liu, S. (2021) Child stunting and land degradation under the Sustainable Development Goals (SDGs): Evidence from 23 developing countries. Land Science, 3(1) 15-21. https://doi.org/10.30560/ls.v3n1p15

16. Liu, S. (2020). Interlinkages between indicators of Sustainable Development Goals: Evidence from seven low income and lower middle-income countries. Sustainable Development Research, 2(1), 58-63. https://doi.org/10.30560/sdr.v2n1p58

17. Lucas, P.L., Hilderink, H.B.M., Janssen, P.H.M. et al (2019) Future impacts of environmental factors on achieving the SDG target on child mortality-A synergistic assessment. Global Environmental Change. 57: 101925. https://doi.org/10.1016/j.gloenvcha.2019.05.009

18. Mirza, M.U., Richter, A., van Nes, E.H. et al (2019) Technology driven inequality leads to poverty and resource depletion. Ecological Economics, 160: 215-226. https://doi.org/10.1016/j.ecolecon.2019.02.015

19. Nilsson, M., Griggs, D. and Visbeck, M. (2016). Policy: Map the interactions between Sustainable Development Goals. Nature, 534(7607): 320-322. doi: https://doi.org/10.1038/534320a

20. Qiu, G., Yin, J. and Geng, S. (2012) Impact of climate and land-use changes on water security for agriculture in Northern China. Journal of Integrative Agriculture. 11: 144-150. https://doi.org/10.1016/S1671-2927(12)60792-5

21. Shaw, S., Khan, J. and Paswan, B. (2020) Spatial modeling of child malnutrition attributable to drought in India. International Journal of Public Health. 65 (3), 281-290. Doi: https://doi.org/10.1007/s00038-020-01353-y

22. Suárez-Varela, M., Esteve, P., Blanco-Gutiérrez, I. et al (2018) Effects of water stress on food security and socioeconomic development. Project working paper. www.madforwater.eu

23. United Nations (2021) SDG indicators: United Nations global SDG database (latest update April 2021). https://unstats.un.org/sdgs/indicators/database/

24. Vanham, D., Hoekstra, A.Y., Wada, Y. et al (2018) Physical water scarcity metrics for monitoring progress towards SDG target 6.4: An evaluation of indicator 6.4.2 "Level of water stress". Science of the Total Environment. 613-614: 218-232. http://dx.doi.org/10.1016/j.scitotenv.2017.09.056

25. Vanham, D. and Leip, A. (2020) Sustainable food system policies need to address environmental pressures and impacts: The example of water use and water stress. Science of the Total Environment. 730:139151. https://doi.org/10.1016/j.scitotenv.2020.139151 
26. Wang, D., Hubacek, K. Shan, Y. et al (2021) A Review of Water Stress and Water Footprint Accounting. Water. 13: 201. https://doi.org/10.3390/w13020201

27. World Bank (2021) Data Bank: Metadata Gsary. https://databank.worldbank.org/metadataglossary/world-development-indicators/series/E R.H2O.FWST.ZS (CC BY-NC-ND) license (https://creativecommons.org/licenses/by-nc-nd/4.0). 